\title{
La llegada de las nuevas tecnologías a la educación y sus implicaciones
}

Recibido: 7 de octubre de 2019 / Revisado: 11 de octubre de 2019

Aceptado: 29 de octubre de 2019 / Publicado: 20 de diciembre de 2019

CYNTHIA CALERO SÁNCHEZ

Facultad de Ciencias de la Educación,

Universidad de Granada, España

cynthiacs@correo.ugr.es

http://dx.doi.org/10.24310/IJNE2.2.2019.7449

\section{RESUMEN}

En las últimas décadas hemos sido testigos del proceso de integración de las nuevas tecnologías a la educación. La llegada de las TIC a las aulas ha traído consigo ciertas ventajas, pero también algunos inconvenientes que nos obligan a interrogarnos acerca de la relación entre la incorporación de las nuevas tecnologías y la mejora de la calidad educativa.

Así, la integración de las nuevas tecnologías a la educación ha supuesto cambios en el currículo, en los centros educativos y sus instalaciones, en los roles del alumnado y el profesorado, etc. Ahora bien, la realidad actual de las aulas demuestra que en su mayoría las nuevas tecnologías han implicado una innovación tecnológica (uso de proyectores, plataformas virtuales, recursos y materiales didácticos, etc.), pero no ha ido acompañada de una innovación pedagógica.

Ahora bien, la consecuencia más relevante del proceso de integración de las TIC a las aulas y que aún constituye una asignatura pendiente para nuestra educación es la posibilidad de poner en marcha in-

\section{ABSTRACT}

The arrival of new technologies to education and their implications

In recent decades we have observed a process of integrating new technologies into education. The arrival of ICT in the classroom has brought with it certain advantages, but also some inconveniences that force us to question ourselves about the relationship between the incorporation of new technologies and the improvement of educational quality.

Therefore, the integration of new technologies into education has resulted in changes in the curriculum, in schools and their facilities, in the roles of students and teachers, etc. However, the current reality of the classrooms shows that most of the new technologies have involved a technological innovation (use of projectors, virtual platforms, resources and teaching materials, etc.), but has not been accompanied by a pedagogical innovation.

However, the most relevant consequence of the process of integrating ICTs into classrooms and which 
novaciones educativas, dando lugar a un proceso de enseñanza-aprendizaje constructivista, activo, colaborativo y personalizado. Un proceso de enseñanzaaprendizaje diseñado, tutorizado, mediado, guiado y evaluado por el profesorado, pero cuyo principal protagonista sea el alumnado.

Palabras Clave: calidad educativa, competencia digital, educación, Tecnologías de la Información y la Comunicación (TIC). still constitutes a pending subject for our education is the possibility of launching educational innovations, leading to a constructivist, active, collaborative and personalized teaching-learning process. A teachinglearning process designed, tutored, mediated, guided and evaluated by teachers, but whose main protagonist is the student.

Keywords: educational quality, digital competence, education, Information and Communication Technologies (ICT).

\section{Introducción}

La sociedad actual no puede entenderse sin la presencia de las nuevas tecnologías, esto es, los últimos desarrollos tecnológicos y sus aplicaciones. Su intervención ha conquistado todas las esferas de nuestra vida, incluido el ámbito de la educación, dando lugar a un nuevo paradigma educativo que trata de dar respuesta a las nuevas demandas sociales de un mundo global, multicultural e interconectado. Sin embargo, cabría preguntarnos: ¿mejora la integración de las nuevas tecnologías la calidad de la educación?

El objetivo de este artículo es precisamente investigar acerca de la integración de las nuevas tecnologías a la educación: las causas y el contexto de la integración, las ventajas y desventajas de este proceso y sus consecuencias.

En esta investigación, por tanto, nuestro objeto de estudio serán las nuevas tecnologías y su integración en el ámbito educativo español, prestando especial atención a las particularidades de la Comunidad Autónoma de Andalucía.

De este modo, expondremos en primer lugar el paulatino proceso de integración, dividido en tres etapas reguladas institucionalmente por diversos planes europeos, nacionales y autonómicos. Además, describiremos los requisitos necesarios para dicho proceso y las dificultades que se detectaron en el mismo. A continuación, analizaremos las ventajas y desventajas derivadas de la integración de las TIC en las aulas, así como los cambios que estas nuevas tecnologías han supuesto en los roles del profesorado y del alumnado. 


\section{Material y Método}

De este modo, para estudiar este proceso de integración de las nuevas tecnologías a la educación se ha llevado a cabo una revisión bibliográfica de obras y artículos publicados desde finales del siglo XX - momento en el que se inició la preocupación por la investigación en tecnología educativa en nuestro país - hasta la actualidad.

La investigación educativa es aquella actividad científica cuyo objetivo es aportar explicaciones razonables de los fenómenos estudiados que contribuyan a la creación de conocimientos que permitan introducir mejoras en la educación (Tejedor, 2008, pág. 177).

En las dos últimas décadas, los trabajos de investigación en tecnología educativa se han incrementado notablemente y en ellos podemos identificar diferentes líneas de investigación como, por ejemplo: investigaciones sobre los medios desde un punto de vista técnico (Bartolomé, A. R. et al, 1989); investigaciones sobre el análisis de los medios con un enfoque didáctico-curricular desde la perspectiva del profesor (Cebrián, 2005); investigaciones sobre el uso de las TIC en los centros educativos y sus efectos en el aprendizaje (Area, 2002); etc.

Cabe destacar que durante mi investigación he procurado conjugar los discursos historiográficos con la referencia a proyectos educativos y la aportación de datos estadísticos y resultados de trabajos de campo.

\section{Resultados}

\subsection{El proceso de integración de las TIC a la educación}

Llamamos integración al "proceso complejo de asimilación y acomodación entre las nuevas tecnologías y el sistema escolar" (Rodríguez, J. L. et al., 1995, pág. 402). La integración de las TIC a los centros educativos ha sido un proceso lento y complejo que se inició en la década de los ochenta y que, a día de hoy, sigue sin haberse completado. Dicho proceso puede dividirse para el contexto español en tres fases (Area, 2002) que exponemos a continuación.

La primera fase, en la década de los ochenta, estuvo regulada institucionalmente por el Proyecto "Atenea", impulsado por el Ministerio de Educación y Ciencia, que creó el "Programa de Nuevas Tecnologías de la Información y Comunicación” (PNTIC). Paralelamente, comenzaron a aparecer en las distintas Comunidades Autónomas planes dirigidos a impulsar el uso de los ordenadores con fines educativos como el Plan "Zahara" en Andalucía. 
Esta primera fase de experimentación consistió principalmente en incorporar la informática como asignatura en los planes de estudio, dotar a los centros de los recursos tecnológicos y utilizar los ordenadores en las tareas de gestión administrativa de los centros educativos. Sin embargo, pese a lo prometedor de los planes, esta etapa estuvo marcada por la incapacidad de los centros educativos para afrontar los costes económicos necesarios para adquirir los recursos tecnológicos.

Debido a los problemas económicos mencionados, la segunda fase, en la década de los noventa, estuvo caracterizada por el estancamiento o desaparición de los programas mencionados y la aplicación de las tecnologías a la educación dejó de ser una prioridad para las administraciones. Los planes fracasaron ya que el uso de los ordenadores seguía siendo muy reducido y no habían logrado mejorar o innovar los procesos de enseñanza-aprendizaje.

Esta situación cambió con la llegada del Congreso Europeo en Barcelona en 1992 sobre las "Tecnologías de la Información y Comunicación. Una visión crítica”, que reflexionó acerca del posible uso de las nuevas tecnologías para mejorar la educación. Este Congreso tuvo lugar en un contexto de revolución tecnológica caracterizado por la generalización de la telefonía móvil, el acceso a internet, la creación de servicios online, etc. que invadió todos los aspectos de la vida cotidiana.

Poco más tarde, en el año 2000 tuvo lugar un Consejo Europeo en Lisboa que estableció como objetivo "convertir a Europa en la economía más competitiva y dinámica del mundo con las aportaciones de las TIC” (Gallego M. J., 2013, pág. 38). A esta iniciativa europea se unieron otras como el propósito de la "Cumbre Mundial sobre la Sociedad de la Información" (CMSI) de la UNESCO celebrada en Ginebra en 2003, que pretendía reducir la brecha digital global; o el informe de la Organización para la Cooperación y el Desarrollo Económico (OCDE) en 2002 titulado Los desafíos de las tecnologías de la información y las comunicaciones en la educación, que identifica una serie de directrices para incorporar las TIC a la realidad educativa (formación del profesorado, cambios tecnológicos de los centros, etc.) (Gallego M. J., 2013, págs. 35-43).

A tenor de las exigencias europeas, en el contexto español se comenzaron a impulsar nuevamente programas destinados a regular y facilitar la integración de las TIC al ámbito escolar. Por ejemplo, en 1999 se pone en marcha el "Plan Info XXI: La sociedad de la información para todos (2000-2003)", que pretendía llevar las tecnologías a todas las ciudades, especialmente en los ámbitos de la sanidad, la educación y la administración.

En 2005 el Ministerio de Industria, Turismo y Comercio crea el "Plan Avanza", una apuesta del gobierno por el desarrollo de la Sociedad de la Información y del Conocimiento. Este plan se continúa en 2011, en una coyuntura de crisis económica, bajo el nombre del "Plan Avanza 2", con el fin de fomentar la recuperación económica a partir del uso y generalización de las TIC en sectores estratégicos como el industrial. 
A nivel educativo, el gobierno central lanza en 2009 el proyecto "Escuela 2.0", en el que participan la mayor parte de las Comunidades Autónomas, exceptuando Madrid y Valencia. El proyecto tiene como objetivo fundamental la modernización del sistema educativo mediante la integración de las TIC. Para ello se propone dotar de recursos TIC a los centros educativos; asegurar la conectividad a Internet dentro y fuera del aula; velar por la formación tecnológica del profesorado; generar y facilitar el acceso a materiales digitales educativos; e implicar al alumnado y sus familias en la custodia y uso de estos recursos (Gallego M. J., 2013, págs. 43-49).

Al mismo tiempo, las administraciones educativas de las diferentes Comunidades Autónomas han propuesto programas propios para la integración de las tecnologías digitales en los centros educativos como, por ejemplo, el proyecto “Averroes" en Andalucía.

En el caso concreto de Andalucía, "es de las Comunidades Autónomas que ha seguido más a rajatabla el "Programa Escuela Tic 2.0" (denominación autonómica de "Escuela 2.0")". De hecho, se han creado blogs y páginas web por iniciativa del profesorado para ofrecer recursos TIC innovadores para las aulas. Además, la página web de la Junta de Andalucía posee un apartado informativo acerca de la "Escuela Tic 2.0." con manuales, preguntas frecuentes, enlaces a recursos educativos, etc. (Martín, S. \& Scopeo, O., 2011).

Ahora bien, todos estos programas también han estado sujetos a críticas. En primer lugar, se pone en tela de juicio la falta de coordinación y de colaboración entre los programas autonómicos y los impulsados por el gobierno central. Se plantea así la posibilidad de un plan global que coordine el proceso de integración de las TIC a la educación, exclusivo para el conjunto del país.

Asimismo, gracias a estos planes se ha logrado que, en 2009, el 99.7\% de las escuelas españolas tuvieran acceso a Internet (Gallego M. J., 2013, pág. 43), sin embargo, continúa siendo deficiente la formación de los docentes, la creación de materiales educativos, el apoyo institucional ofrecido por la administración, etc.

De este modo, las TIC se han ido integrando paulatinamente a la educación bajo dos premisas: la primera, que la escuela no puede quedar al margen de las transformaciones de la sociedad y la tecnología; y, la segunda, que estas nuevas tecnologías pueden mejorar la calidad de la enseñanza y posibilitar la innovación de los métodos y materiales didácticos, aunque, como veremos, la presencia per se de las TIC no es garantía de mejora de los procesos de enseñanza-aprendizaje (Barberá, E. et al., 2008, pág. 9).

\subsection{Requisitos}

Ahora bien, para que pueda darse esta integración de las TIC a la educación es necesario cierto esfuerzo económico, técnico y humano que explicamos a continuación. En primer lugar, como hemos 
visto, resulta fundamental la presencia de un plan institucional que regule la incorporación de las nuevas tecnologías a la educación. Pero, sin duda, el primer requisito es disponer de los recursos necesarios para poder introducirlos, hecho que supone una importante inversión económica. En ocasiones "este factor puede determinar la calidad y cantidad de resultados que se obtengan de la introducción de las TIC" (Cebrián, M. \& Gallego, M. J., 2011, pág. 35).

Los centros educativos deben modificar sus estructuras internas para posibilitar el trabajo en red: conexión a internet, dotación y renovación permanente de equipos y programas (hardware y software), etc. Todo ello trae consigo una "nueva cultura de trabajo en red", es decir, las posibilidades de compartir recursos, de automatizar las tareas repetitivas, el diseño de nuevos espacios virtuales de gestión y autoaprendizaje, trabajar desde el hogar, conectar con profesionales de otros centros educativos, etc.

Al mismo tiempo, se requiere una nueva concepción de los espacios y de los tiempos de aprendizaje. En lo que se refiere a los espacios, aparecen nuevas áreas de aprendizaje como salas de proyecciones multimedia o bibliotecas digitales. Además, el aprendizaje puede llevarse a cabo en prácticamente cualquier escenario: la escuela, la universidad, el hogar, el trabajo, el espacio de ocio, etc. En cuanto al tiempo, trata de optimizarse por medio de espacios virtuales en los que se reducen los procesos mecánicos y repetitivos. "Las nuevas tecnologías fomentan la interacción y el aprendizaje sobre todo fuera de las barreras usuales del espacio y el tiempo, y la enseñanza y el aprendizaje se producen en un "no lugar" denominado ciberespacio" (Cebrián, M. \& Gallego, M. J., 2011, pág. 35).

Estos recursos deben ir necesariamente acompañados de la formación tecnológica constante y actualizada del profesorado. Además, se hace necesaria la presencia en la estructura organizativa de los centros educativos del coordinador o coordinadora TIC para el desempeño de tareas como el mantenimiento de los recursos tecnológicos, la gestión de su uso, el asesoramiento al profesorado, etc. (Fernández, M.C. \& Cebreiro, B., 2003).

Sin embargo, no solo se demandan cambios tecnológicos y de las infraestructuras, sino también un cambio de mentalidad que reconozca las nuevas formas de producir conocimiento y las nuevas posibilidades de aprender que ofrecen las nuevas tecnologías. Así, necesariamente se tienen que modificar los procesos de enseñanza-aprendizaje, ahora más centrados en el alumnado, con un rol más activo y autónomo, y mediados por el profesorado.

La integración de la TIC también afecta al currículo, que debe ser capaz de flexibilizarse para incluir la presencia de los recursos tecnológicos, no solo como medios de aprendizaje, sino también como motores de un cambio didáctico. 


\subsection{Dificultades}

Ahora bien, no son pocas las dificultades presentes en el proceso de integración de las TIC a la educación. Entre estas dificultades podemos mencionar: problemas estructurales como la falta o inadecuación de los medios de instalaciones, los costes elevados, la ausencia de mantenimiento del equipamiento; problemas actitudinales como la resistencia a la incorporación de las TIC, el desconocimiento por parte del profesorado acerca de cómo implementar las TIC en su práctica docente; problemas formativos como la insuficiente preparación del profesorado en el uso fructífero de las TIC; etc. (Medina, 2016, pág. 212; Barroso, J. \& Cabero, J., 2015, pág. 57).

No obstante, el error más significativo que se ha cometido en la incorporación de las TIC en los procesos de enseñanza-aprendizaje ha sido implementar los medios tecnológicos sin tener en cuenta otras variables curriculares como las metodologías, los contenidos, los objetivos, etc. No basta con incorporar tecnologías al centro para obtener logros significativos en el aprendizaje del alumnado. "El cambio tecnológico, si no está fundamentado en la metodología didáctica y la formación, es un sinsentido" (Herrán, A. \& Fortunato, I., 2017).

No se trata de introducir las TIC en las escuelas como abordaje utilitario o tecnócrata, atendiendo a la imperiosa necesidad de modernizarse, como panacea del progreso o para responder a las exigencias de los intereses económicos y comerciales que se benefician de la venta de los medios tecnológicos, sino de que se integren las tecnologías con un fin y valor didáctico (Díaz, 2009, pág. 153).

En definitiva, en este proceso de integración de las TIC a la educación hemos completado la fase de equipamiento informático y de conexión a la red en la mayoría de los centros, hemos procedido a la formación para el uso correcto de las nuevas tecnologías, pero ahora falta integrar la informática en el aprendizaje, proceder a un eficaz "aprendizaje con ordenadores" (Tamargo, 2016).

\subsection{Ventajas y desventajas de la llegada de las nuevas tecnologías a la educación}

La puesta en marcha de este proceso de integración de las nuevas tecnologías en la educación ha permitido a diversos investigadores e investigadoras (Cebrián, M. \& Gallego, M. J., 2011; Fajardo, 2010; Roldán, 2015; entre otros) analizar las ventajas y desventajas que ha traído consigo la llegada de las TIC a las aulas.

En primer lugar, en lo que se refiere a las ventajas, debemos señalar la alfabetización digital del alumnado, que como ya hemos descrito con anterioridad, resulta indispensable para desenvolverse en la sociedad y en el mundo laboral actuales. De lo contrario, el alumnado podría verse inmerso en lo que se conoce como "brecha digital", una situación de exclusión social debido a su analfabetismo tecnológico y a la imposibilidad de acceder a los recursos. 
Cuando hablamos de alfabetización digital nos referimos a competencias básicas en el uso de las TIC como: el conocimiento de los sistemas informáticos (hardware, software, redes); el uso del sistema operativo; la búsqueda y selección de información a través de internet; la comunicación interpersonal y el trabajo colaborativo en redes; el procesamiento de textos; el tratamiento de la imagen; la utilización de la hoja de cálculo; el uso de una base de datos; la actitud crítica ante las TIC; etc. (Marqués, 2013).

En segundo lugar, la integración de las nuevas tecnologías en la educación supone el aprovechamiento de sus funciones como fuente abierta de información global; herramienta para procesar y transformar información; instrumento para la gestión administrativa y tutorial; mecanismo de almacenamiento; medio de expresión didáctico y lúdico; canal de comunicación, colaboración e intercambio; etc. (Rosendo, 2010, pág. 16).

Todas estas funciones, en las cuales incidiremos más adelante, permiten aumentar la productividad de todos los miembros de la comunidad educativa, por ejemplo, facilitando la comunicación y la difusión de la información entre profesorado-tutores-alumnado a través de nuevos canales de comunicación como el correo electrónico, las wikis o los foros.

Así, el uso de las nuevas tecnologías en el aula favorece un aprendizaje activo, una mayor interacción e implicación del alumnado en su proceso de aprendizaje. "Las personas que desean aprender se hacen verdaderas protagonistas de su propio aprendizaje” (Roldán, 2015, pág. 46). El empleo de las nuevas tecnologías permite un aprendizaje individual y personalizado. Cada alumno o alumna puede adecuar las herramientas y medios disponibles a su ritmo de trabajo, su estilo de aprendizaje y nivel de conocimiento, evitando las posibles lagunas de aprendizaje que suelen producirse en una clase tradicional y posibilitando una mayor atención a la diversidad. En lo que se refiere a esto último, las nuevas tecnologías ofrecen herramientas, aplicaciones, etc. para solventar las posibles dificultades visuales, auditivas, etc. del alumnado con necesidades educativas especiales. El ordenador, además, tiene el estimulante de integrar al alumnado con necesidades educativas especiales entre sus compañeros porque los medios que utilizan son aparentemente iguales.

Ahora bien, pese a que el uso de las nuevas tecnologías hace que el proceso de enseñanza-aprendizaje sea más personal e individualizado, también favorece la colaboración con los compañeros del aula e incluso con miembros ajenos al centro debido a la eficacia de las redes sociales. En este sentido, las TIC favorecen el aprendizaje colaborativo y facilitan la comunicación entre el alumnado gracias a herramientas como Google Docs o One drive, que reducen los inconvenientes a la hora de reunirse, independientemente de su situación geográfica y la disponibilidad temporal.

Además, se ha demostrado que el empleo de las nuevas tecnologías configura otros modos de expresarnos, de aprender y de conocer que mejoran la capacidad de comprensión y el tratamiento de 
la información digital, así como su representación, no solo textual, sino también a través de imágenes, textos, vídeos, sonidos, animaciones, representaciones virtuales en 3D, realidad aumentada, etc. "La palabra docente y el texto escrito dejan de ser los únicos soportes de la comunicación educacional" (Fajardo, 2010). De este modo, este variado e interactivo lenguaje multimedia aumenta la retención de los conceptos por parte del alumnado y facilita la comprensión de los contenidos debido a su mayor similitud y cercanía con la realidad. Además, estos contenidos representados resultan más atractivos y entretenidos para el alumnado, que se muestra más motivado por aprender (Cebrián, M. \& Gallego, M. J., 2011, pág. 24).

En lo que se refiere a las ventajas en la práctica docente, gracias al uso de las nuevas tecnologías, el profesorado tiene a su disposición gran cantidad de información, recursos, aplicaciones, programas, etc. para diseñar, exponer y evaluar en sus clases. Dicho de otro modo, las posibilidades didácticas que ofrecen las TIC permiten innovar en las prácticas docentes, mejorar los procesos de aprendizaje y, en consecuencia, reducir el fracaso escolar.

Otra diferencia significativa con respecto a la clase tradicional es la posibilidad de una "consistencia pedagógica" (Rosendo, 2010, pág. 29). Esto quiere decir que, con la introducción de las nuevas tecnologías, la lección, los contenidos, las actividades, etc. pueden repetirse de manera idéntica las veces que sean necesarias y, en consecuencia, la calidad de la educación y la enseñanza no varía de una clase a otra, ni, si se quiere, de un colegio a otro.

No obstante, la aplicación de las nuevas tecnologías en la educación también conlleva ciertas desventajas o inconvenientes. En primer lugar, la necesidad de recursos económicos de los centros educativos para poder llevar a cabo dicha aplicación. Para que pueda producirse una correcta integración en las TIC en las aulas el centro docente debe contar con: una intranet - o red privada - educativa realmente operativa y ordenadores en buenas condiciones de uso y suficientes para la ratio de alumnos. Asimismo, tanto el alumnado como el profesorado necesita disponer de ordenador y conexión a internet en casa o, en su defecto, que el municipio disponga de áreas que posibiliten el acceso a Internet como bibliotecas, zonas wifi, etc. (Palomo, 2010). Además de estos requisitos previos que pueden suponer un inconveniente para introducir las TIC en las aulas, no debemos olvidar que esta dependencia tecnológica en ocasiones se ve afectada por los problemas de mantenimiento de estos recursos, las dificultades de conexión a la red y otros fallos técnicos posibles.

Además, otro inconveniente podría ser la falta de especialistas informáticos y la necesidad de formación específica y continua entre los docentes y gestores de los centros educativos.

En relación con esto último, parte del profesorado se muestra en ocasiones reticente a integrar las nuevas tecnologías en su labor docente, bien por el esfuerzo formativo que mencionábamos con anterio- 
ridad, bien por considerar que poseen resultados negativos para el alumnado y, en consecuencia, optan por mantener el modelo educativo tradicional.

En efecto, pese a las ventajas enunciadas, la introducción de las nuevas tecnologías podría conllevar efectos peyorativos, por ello es fundamental la mediación del profesorado para tratar de paliarlos.

En primer lugar, el uso continuo de las TIC ha traído consigo la emergencia de nuevos trastornos físicos y psicológicos como degeneraciones de la vista, enfermedades musculares o de las articulaciones, alteraciones del sueño, adicciones a Internet, cambios en la personalidad, etc. (Trujillo, J. M et al., 2013, pág. 155).

En segundo lugar, la reducción de la intimidad y privacidad que frecuentemente aparecen asociados al uso de las nuevas tecnologías implica un creciente nivel de exposición que puede traer problemas a la juventud, poco consciente de los peligros que esto supone. Existe el riesgo de entrar en contacto con personas que influyan negativamente en el alumnado o que se aprovechen del mismo con fraudes, intimidaciones, difusión de datos, etc. Otros usos perversos de las nuevas tecnologías incluyen conductas violentas, agresivas y antisociales como el cyberbullying, el acceso a websites inadecuados según la edad, los fraudes o la piratería (Trujillo, J. M et al., 2013, págs. 155-190).

Según Naciones Unidas, en el Estudio Mundial sobre la Violencia contra los Niños y Niñas de año 2005: "Internet y los teléfonos celulares han proporcionado nuevas oportunidades de acoso o intimidación a través de los mensajes de correo electrónico, los chat, las páginas web personales, los mensajes de texto y la transmisión de imágenes" (Cebrián, M. \& Gallego, M. J., 2011, págs. 62-63). Una violencia con efecto multiplicador, ya que se difunde más allá del espacio reducido del aula y posee una mayor cantidad de receptores.

Por otra parte, las nuevas tecnologías hacen que la escuela deje de ser el único medio que pone en contacto al alumnado con el conocimiento y la información. El alumnado puede acceder a la información fuera de las barreras del aula. Ahora bien, esta información incluye contenidos de corte publicitario, influencias de los gobiernos, las empresas y la sociedad en general que aportan realidades sesgadas y manipuladas que pueden condicionar las opiniones del alumnado y tergiversar o falsear dicha información. Concretamente, el alumnado se encuentra expuesto a contenidos violentos, racistas, machistas, que fomentan el consumo de drogas, la anorexia, entre muchos otros.

No debemos olvidar que las nuevas tecnologías no son inocentes y que, lejos de ser simples recursos educativos, medios transparentes que no presentan ideología alguna, son el reflejo de una cultura determinada (democrática, capitalista, consumista, etc.) con la que el alumnado debe de aprender a ser crítico (Diez, Á. et al., 2003, pág. 36). 
Por otra parte, conviene desmentir algunos mitos vinculados con las TIC. En primer lugar, el considerar que la introducción de las nuevas tecnologías en el aula crea inmediatamente un incremento en los procesos de enseñanza-aprendizaje. Como señalábamos con anterioridad, es un error considerar que la sustitución de los medios antiguos por las nuevas tecnologías implica una mejora educativa. Para ello, este cambio metodológico debe ir acompañado de una propuesta pedagógica y didáctica que explote las potencialidades de las TIC para alcanzar un aprendizaje significativo, activo, constructivo, interactivo, etc. (Cabero, 2006, págs. 207-209)

En segundo lugar, el prejuicio que concibe que las nuevas tecnologías pueden sustituir la labor docente y al profesor. "Esta posición reduce erróneamente el rol del profesor a tecnólogos encargados de utilizar recetas formativas a un determinado conjunto de alumnos" (Rosendo, 2010, págs. 16-17). No obstante, lo que es innegable es que la introducción de las nuevas tecnologías en las aulas, con el cambio en la manera de concebir la educación que esto supone, varía notablemente la manera de enseñar y la manera de aprender, esto es, los roles tanto del profesorado, como del alumnado.

\subsection{El nuevo rol guía-orientador del profesorado}

Frente a todo el entramado tecnológico con el que nos encontramos en la actualidad, el profesorado sigue siendo la pieza clave para hacer que el sistema educativo funcione. "La tecnología sola no garantiza la innovación educativa; se requiere la intervención activa del educador" (Gallego, D. J. et al., 2011, pág. 9). Esto no significa, sin embargo, que el docente no tenga que cambiar sus funciones y roles a desempeñar.

En este nuevo contexto marcado por la presencia de las nuevas tecnologías, "el profesor deja de ser la fuente principal de todo conocimiento, pasando a actuar como guía del alumnado, facilitándoles el uso de los recursos y las herramientas necesarias para explorar y elaborar nuevos conocimientos y destrezas" (Medina, 2016, pág. 213).

De este modo, el profesorado abandona el rol de transmisor y depositario de la información y el alumnado deja de ser mero receptor y reproductor de la información. Este modelo de formación tradicional es sustituido por el aprendizaje constructivista.

En el ámbito de la educación concebimos el constructivismo como el proceso de aprendizaje por el cual el alumno o la alumna construye y reconstruye su propio conocimiento, gracias a la actividad de su sistema nervioso central, teniendo en cuenta los conocimientos previos (Ortíz, 2015). En este proceso influyen las capacidades físicas del sujeto, su estado emocional, sus condiciones sociales y culturales, etc. Y, en el caso de la escuela, intervienen además otros factores como la planificación y estrategias del docente o la motivación. 
A diferencia de la escuela transmisiva, en la que el docente ofrece un conocimiento ya elaborado para que el alumnado lo asimile, el constructivismo se basa en que el alumnado, con un papel activo, construye su conocimiento, reconstruyendo los conocimientos ya adquiridos en un contexto social y cultural determinado. En el aprendizaje constructivista el docente actúa como orientador que guía el aprendizaje del alumno, que media entre los contenidos del aprendizaje y la actividad constructiva del alumno. Para ello el docente evita convertirse en transmisor de la información porque acepta e impulsa la autonomía e iniciativa del alumno y, en lugar de esto, propone y motiva a sus alumnos para resolver problemas/debates/ proyectos con unos determinados materiales, ambos elegidos en función de sus conocimientos previos, su contexto y sus estructuras cognoscitivas con el fin de fomentar un aprendizaje significativo.

A pesar de que el profesorado abandona su papel transmisor, las funciones —asociadas muchas de ellas al uso de las TIC - que desempeña hoy en día son múltiples (Barroso, J. \& Cabero, J., 2015, págs. 59-62). En primer lugar, el profesorado debe seleccionar y adaptar la gran cantidad de información disponible a las necesidades del alumnado. En la Sociedad del Conocimiento, el alumnado puede acceder a la información en cualquier lugar, pero conviene que el profesorado acote previamente dicha información y guíe al alumnado acerca del tratamiento de la misma - cómo buscar información, qué criterios seguir para seleccionarla y sintetizarla, etc. - con el fin de que se convierta en conocimiento. "El alumnado puede informarse dándole a un botón, lo que no puede es educarse dándole a un botón" (Fajardo, 2010), necesita que el profesorado actúe como mediador de las situaciones de aprendizaje.

En segundo lugar, los docentes, aprovechando los recursos y materiales que ofrecen las TIC, diseñarán los medios y los entornos de aprendizaje teniendo como objetivo guiar y facilitar el aprendizaje del alumnado. El profesorado no solo deja de ser mero transmisor de la información, sino que además se encargará de "crear" la información que va a recibir su alumnado. Por tanto, "el rol del educador se ha ampliado y es el eje central en la producción de material educativo" (Gallego, D. J. et al., 2011, pág. 11).

En otras palabras, "el profesor se convertirá en curador de contenidos" (Barroso, J. \& Cabero, J., 2015, pág. 226). En este proceso es importante tener en cuenta que los entornos de aprendizaje estén a disposición de la diversidad del alumnado - más allá de su localización, acceso a la tecnología, ritmo de aprendizaje, necesidades, etc. - que progresivamente irá avanzando en la adquisición de conocimientos y la realización de actividades, hasta llegar a su evaluación.

La introducción de las TIC en las aulas proporciona al profesorado innumerables herramientas que le ayudan a desempeñar su labor docente: recursos, medios, materiales didácticos, programas, etc. para extraer contenidos, diseñar las clases, facilitar el seguimiento del alumnado y su evaluación, etc.

Además, las nuevas tecnologías permiten que el diseño de estos entornos de aprendizaje no sean una actividad docente individual sino colaborativa, en la que pueden participar otros profesionales que 
comparten esfuerzos y experiencias. En consecuencia, otro de los roles fundamentales del profesorado es el trabajo en equipo y el abandono de clases magistrales individuales a puerta cerrada.

Ahora bien, para estos autores (Barroso, J. \& Cabero, J., 2015, pág. 62), los dos roles más significativos del profesorado son el de "moderador y tutor virtual". Estas funciones comprenden tareas como impartir formación sobre el funcionamiento técnico de las nuevas tecnologías; gestionar el trabajo en red del alumnado; animar al estudiantado al autoestudio; supervisar y evaluar el progreso del alumnado en sus procesos de aprendizaje; etc.

En síntesis, "el profesorado deberá facilitar el aprendizaje del estudiantado aprovechando las posibilidades de los medios tecnológicos para que este aprendizaje se produzca en todas sus dimensiones" (Cebrián, M. \& Gallego, M. J., 2011, pág. 27). Para ello, debe ejercer competencias como: asesor y guía para favorecer el aprendizaje; motivador y facilitador de los recursos; diseñador de nuevos entornos de aprendizaje TIC; productor de materiales didácticos; evaluador de los procesos de aprendizaje; etc.

Ahora bien, ninguno de estos roles puede cumplirse sin iniciativa tecnológica, es decir, sin formación continua y permanente en el uso de las TIC. De este modo, la formación del profesorado debe combinar la adquisición de las competencias digitales o destrezas tecnológicas con su aplicación didáctica, es decir, aprender a enseñar con TIC (García, 2013).

Como vemos, la nueva realidad tecnológica exige mucho más al profesorado, "ya de por sí denostado socialmente y colmado de demandas personales, profesionales y mercantiles" (Trujillo, J. M et al., 2013, pág. 205). Los nuevos profesores y profesoras deben asumir competencias profesionales adicionales vinculadas con el manejo de las nuevas tecnologías tales como: manejo de aplicaciones básicas de programas de edición y de publicación de páginas web; conocimientos sobre planificación y organización de las TIC en el aula; capacidad de acceso a las fuentes de información y recursos en soporte TIC; aprovechamiento de los recursos y aplicaciones TIC para el diseño de las intervenciones didácticas y para la evaluación del alumnado; conocimientos sobre los procesos de comunicación que ofrecen las nuevas tecnologías; enseñanza al alumnado de nociones básicas de autoaprendizaje a través de las TIC; etc. (Gallego D. , 2007, págs. 125-126).

Además, entre estas nuevas demandas se encuentra la ampliación del tiempo dedicado a la labor docente, no solo para la formación en materia digital, sino también para la comunicación virtual. Como consecuencia de que los procesos de aprendizaje tengan como nuevo escenario los entornos virtuales, es a través de sus canales de comunicación por los que se relacionan profesorado-alumnado, alumno/aalumno/a, e incluso, tutores-profesorado. Por consiguiente, esta comunicación virtual se mantiene también fuera del aula y del horario lectivo. 
No obstante, pese a los roles del nuevo profesorado descritos, la realidad es bien distinta. Por un lado, existe una queja colectiva acerca de la insuficiente formación en materia digital con la que cuenta el profesorado. Los docentes asisten a cursos de corta duración, casi siempre fuera de sus centros y con una formación escasa a la hora de intentar convertirla en una práctica pedagógica eficiente (Mena, B. et al, 1996, págs. 113-115).

En un estudio realizado en la comunidad autónoma gallega a principios del siglo XXI, las estadísticas señalan que aproximadamente el $80 \%$ del profesorado no está preparado para el manejo técnico de los medios ni su integración curricular (Fernández, M.C. \& Cebreiro, B., 2003). Fruto de ello, son muchos los profesores reacios al empleo de las nuevas tecnologías y, en consecuencia, se introducen de manera escasa, reduciéndose en su mayoría al uso del vídeo, el equipo de sonido y el proyector de diapositivas. Por tanto, los medios se utilizan sobre todo para transmitir los contenidos, captar la atención y motivación del alumnado y permitir acceso a más información. Sin embargo, se emplean mucho menos para otras funciones como la relación profesorado-alumnado y la evaluación de conocimientos.

\subsection{El alumnado como protagonista}

Por otra parte, la llegada de las nuevas tecnologías a la sociedad ha contribuido a perfilar nuevos modelos de conducta. Los adolescentes en la actualidad se caracterizan por vivir en un mundo interconectado, con facilidad para acceder a grandes cantidades de información, en constante cambio y sometido a varias actividades simultáneas (leer, escuchar música, 'whatsappear' ...), la multitarea. Todo ello dificulta la capacidad de concentración, el interés y la atención hacia lo real - o no virtual - y hacia el estudio por parte del alumnado.

La sociedad actual demanda un cambio en el sistema educativo que sustituya el modelo de enseñanza tradicional por un proceso de aprendizaje en el que el alumnado posea un rol más activo y participativo. La introducción de las nuevas tecnologías en las aulas facilita este reto de la educación, ya que posibilita un aprendizaje más individualizado por parte del alumnado, adecuado a sus conocimientos previos y a su ritmo de trabajo.

El objetivo de la educación es formar al alumnado para que viva en sociedad y hoy en día se requiere un aprendizaje a lo largo de toda la vida. Los cambios son muy vertiginosos y la información a la que accedemos hoy puede estar desfasada mañana. En consecuencia, la prioridad es “conjugar la capacidad cognitiva con la capacidad para aprender, desaprender y reaprender para adaptarse a las nuevas exigencias de la sociedad; esto es, estar capacitado para el autoaprendizaje” (Barroso, J. \& Cabero, J., 2015, págs. 63-64). 
Para ello, el alumnado debe aprender a buscar información, seleccionar la más relevante, sintetizarla, almacenarla, organizarla y hacerla significativa, es decir, convertirla en conocimiento, comprenderla. Todo ello haciendo un uso correcto, crítico y responsable de las TIC (Cebrián, M. \& Gallego, M. J., 2011, pág. 26).

Aunque los alumnos son "nativos digitales", las investigaciones están demostrando que "si bien son bastante competentes para desenvolverse instrumentalmente con las tecnologías, no lo son tanto cuando se trata de utilizarlas en procesos de enseñanza-aprendizaje, seleccionando y evaluando información pertinente y relevante, realizando acciones de aprendizaje colaborativo y gestionando el tiempo que invierten en su interacción" (Barroso, J. \& Cabero, J., 2015, pág. 66). Es aquí donde interviene la figura guía y mediadora del docente para asegurarse de que el alumnado realiza un uso serio y provechoso de las nuevas tecnologías para el aprendizaje y no simplemente la consulta de páginas web para su posterior "copia y pega" en un programa de edición, sin sacarle provecho alguno a la herramienta y a la información (Rosendo, 2010, pág. 18),

Además del desarrollo de habilidades para el autoaprendizaje, el tratamiento de la información y el control de las nuevas tecnologías, otro de los roles que se exigen al alumnado es el trabajo en equipo. En este rol de cooperación se incluyen habilidades como el diálogo, la escucha, la participación activa y la flexibilidad. En la actualidad, en el proceso de construcción del conocimiento es fundamental el trabajo en equipo porque es imposible que una persona pueda aunar toda la información y, por tanto, el trabajo es más rico cuando se reúnen los conocimientos y habilidades de todos los miembros de un equipo.

De la misma manera, la sociedad actual demanda del alumnado roles y habilidades que las nuevas tecnologías no pueden ofrecer como la creatividad, la capacidad de expresión, el sentido crítico y la resolución de problemas (Barroso, J. \& Cabero, J., 2015, pág. 65).

\section{Discusión}

Según el informe del proyecto TICSE 2.0., una investigación sobre el uso de las TIC en las prácticas de enseñanza-aprendizaje en el aula, "los materiales didácticos tradicionales - libros de texto, pizarras, etc. - siguen siendo los recursos más empleados en las aulas Escuela 2.0 a pesar de la abundancia de la tecnología digital" y la mayoría del profesorado afirma que gran parte de las actividades que desarrolla en el aula con TIC se enmarcan dentro de un modelo de enseñanza tradicional (TICSE, 2011, pág. 99).

1 Término acuñado por Prensky (Prensky, 2001) para hacer referencia a las diferencias en el manejo de las nuevas tecnologías entre las personas que han crecido en la red y los que provenían de otras tecnologías como las analógicas ("emigrantes digitales"). 
Como dirían, Adell, J. y Castañeda, L., "tecnología que viste de modernidad un enfoque didáctico tradicional” (Adell, J. \& Castañeda, L., 2012).

En efecto, el principal reto al que se enfrenta el proceso de integración de las TIC a la educación en la actualidad es lograr que la incorporación de las nuevas tecnologías vaya acompañada de una mejora de los procesos de enseñanza-aprendizaje. Este reto se resiente debido a la persistencia del modelo de enseñanza tradicional, la falta de destrezas tecnológicas por parte del profesorado, la ausencia de una modificación curricular y el desarrollo deficitario de la infraestructura y recursos tecnológicos en los centros educativos, debido a las limitadas inversiones económicas. "El reto a medio plazo de los programas y proyectos institucionales, en consecuencia, debiera ser la innovación y cambio pedagógico del sistema escolar y de la cultura organizativa de los centros, no solo la dotación de recursos tecnológicos a las aulas y centros" (Area, 2002).

En otras palabras, ni la incorporación ni el uso de las TIC implica automáticamente la transformación, innovación y mejora de las prácticas educativas. Sin embargo, las TIC abren la posibilidad de generar nuevas formas de aprender y enseñar, de poner en marcha procesos de enseñanza-aprendizaje que no serían posibles en ausencia de las TIC (Coll, 2009).

\section{Conclusiones}

En conclusión, la integración de las nuevas tecnologías a la educación no mejora sin más la calidad del proceso de enseñanza-aprendizaje, pero sí abre la posibilidad de llevar a cabo una innovación pedagógica. Para ello es indispensable, no solo la disponibilidad de medios tecnológicos, la formación digital por parte del profesorado y el compromiso de la comunidad educativa, sino también una transformación en la concepción del aprendizaje tal y como lo concebimos hasta el momento. Las TIC permiten el desarrollo de un nuevo aprendizaje activo, colaborativo, abierto, personalizado.

La puesta en marcha de esta innovación pedagógica, sin embargo, necesita de una importante implicación en tiempo y en esfuerzo por parte del profesorado, una destacable inversión por parte del centro educativo y cambios en el currículo que flexibilicen la concepción del aprendizaje.

Con sus ventajas y sus desventajas, inevitablemente, las nuevas tecnologías ya están aquí, en las aulas, porque la educación no puede vivir ajena a la realidad social que la rodea, con unos modelos educativos decimonónicos dirigidos a unos estudiantes nativos digitales, que cada vez permiten entrever mayores problemas de concentración y de atención. Por el contrario, la educación está llamada a motivar a su alumnado y a incorporar medios como las nuevas tecnologías que contribuyan a, primero, formar a los estudiantes en las competencias que desarrollarán en el futuro como ciudadanos y profesionales 
- competencia digital, espíritu crítico, trabajo en equipo, etc. - y, segundo, a favorecer el desarrollo de innovaciones pedagógicas que mejoren el aprendizaje.

De nada sirve sustituir los libros de texto por portátiles si las clases siguen estando dominadas por un profesorado que expone los contenidos y un alumnado pasivo que escucha, memoriza y se examina. Es el momento de ceder el papel activo a un alumnado que deberá adquirir las habilidades necesarias para aprender de manera autónoma a lo largo de la vida, gracias a la guía, tutorización y orientación del profesorado. Aun así, para que el profesorado pueda jugar ese papel necesita recibir una formación adecuada en cuanto a la competencia digital y el aprovechamiento didáctico de las TIC.

Por otra parte, las nuevas tecnologías tienen la capacidad de revolucionar los procesos de enseñanza-aprendizaje aportando innumerables recursos y materiales didácticos útiles tanto para el alumnado como el profesorado. Nunca antes el alumnado pudo aprender de una manera tan dinámica, interactiva y realista; ni el profesorado tuvo a su alcance tantos medios para diseñar, gestionar y evaluar sus clases.

En síntesis, las nuevas tecnologías ya han arribado a las aulas, pero la innovación pedagógica aún está por llegar.

\section{Referencias}

Adell, J. \& Castañeda, L. (2012). Tecnologías emergentes, ¿pedagogías emergentes? En J. Hernández et al., Tendencias emergentes en educación con TIC (págs. 13-32). Barcelona: Espiral.

Area, M. (2002). La integración de las nuevas tecnologías. Entre el deseo y la realidad. Revista del Fórum Europeo de Administradores de la Educación, 10(6), 1418.

Barberá, E. et al. (2008). Cómo valorar la calidad de la enseñanza basada en las TIC. Pautas e instrumentos de análisis. Barcelona: Graó.

Barroso, J. \& Cabero, J. (2015). Nuevos retos en tecnología educativa. Madrid: Editorial Síntesis.

Bartolomé, A. R. et al. (1989). Nuevas tecnologías y enseñanza. Barcelona: Graó.

Cabero, J. (2006). Nuevas Tecnologías Aplicadas a la Educación. Madrid: Mc Graw Hill.

Cebrián, M. \& Gallego, M. J. (2011). Procesos educativos con TIC en la sociedad del conocimiento. Madrid: Ediciones Pirámide. 
Cebrián, M. (2005). Tecnologías de la información y comunicación para la formación de docentes (Vol. 20). Madrid: Ediciones Pirámide.

Coll, C. (2009). Aprender y enseñar con las TIC: expectativas, realidad y potencialidades. En R. Carneiro et al., Los desafíos de las TIC para el cambio educativo (págs. 113-126). Madrid: Santillana.

Díaz, F. (2009). TIC y competencias docentes del siglo XXI. En R. Carneiro et al., Los desafíos de las TIC para el cambio educativo (págs. 139-154). Madrid: Santillana.

Diez, Á. et al. (2003). Nuevas tecnologías, educación y sociedad. Perspectivas críticas. Cuadernos de Trabajo de Hegoa (36).

Fajardo, F. (2010). Influencia de las tecnologías de la información y la comunicación en la educación. Tejuelo, Monográfico 4, 9-17.

Fernández, M.C. \& Cebreiro, B. (2003). La integración de los medios y nuevas tecnologías en los centros y prácticas docentes. Pixel-Bit. Revista de Medios y Educación, 20, 33-42.

Gallego, D. (2007). Profesión y Docencia. El Nuevo Perfil de la Profesión Docente. Informe 2007. En M. Juste et al., Actas del IV Congreso Internacional de EDUCARED: Educar en Comunidad (págs. 105-132). Madrid: Fundación Telefónica.

Gallego, D. J. et al. (2011). Educación, sociedad y tecnología. Madrid: Editorial Universitaria Ramón Areces.

Gallego, M. J. (2013). Aplicaciones de las TIC en contextos educativos. Líneas y ámbitos de trabajo. Barcelona: Davinci Continental.

García, D. M. (2013). Estilos de enseñanza y las nuevas tecnologías en la educación. ReiDoCrea. Revista electrónica de investigación Docencia Creativa, 2, 219-225.

Herrán, A. \& Fortunato, I. (2017). La clave de la educación no está en las nuevas Tecnologías de la Información y la Comunicación (TIC). Acta Scientiarum. Education, 39(3), 311-317.

Marqués, P. (2013). Impacto de las TIC en la educación: funciones y limitaciones. 3C TIC, 2(1).

Martín, S. \& Scopeo, O. (2011). Escuela 2.0.: panorama actual de la situación del programa. Ponencia presentada en el X Seminario del Consejo Escolar de Castilla-León. Red XXI: Una puerta a la educación del futuro. Obtenido de http://scopeo.usal.es/wp-content/ uploads/2012/10/Ponencia escuela2.0.pdf 
Medina, I. D. (2016). La inclusión de las TIC en las enseñanzas medias. Un estudio de caso. En I. A. Sánchez, Del individuo al aprendizaje colaborativo (II). La historia y la historia del arte frente a las salidas profesionales del mundo laboral, en el contexto educativo y la gestión de la información (págs. 209-225). Málaga: ExLibric.

Mena, B. et al. (1996). Didáctica y nuevas tecnologías en educación. Madrid: Escuela Española.

Ortíz, D. (2015). El constructivismo como teoría y método de enseñanza. Sophia: colección de Filosofia de la Educación, 19(2), 93-110.

Palomo, J. A. (2010). La Web 2.0.: una aplicación didáctica para las ciencias sociales. Tejuelo, Monográfico 4, 18-31.

Prensky, M. (2001). Digital natives, digital inmigrants. On the horizon, 9(5), 1-6.

Rodríguez, J. L. et al. (1995). Tecnología educativa: nuevas tecnologías aplicadas a la educación. Alcoy: Marfil.

Roldán, L. (2015). Un ejemplo de innovación educativa en Historia: la utilización de blogs como estrategia de aprendizaje y evaluación. En J. A. Sánchez, Del individuo al aprendizaje colaborativo (I). La Historia y la Historia del Arte ante los retos de la innovación educativa (págs. 43-54). Málaga: ExLibric.

Rosendo, D. (2010). Las ciencias sociales y las nuevas tecnologías de la educación. Sevilla: Wanceulen Editorial.

Tamargo, L. Á. (2016). Las TIC en la educación: previsiones a corto, mediano y largo plazo. En M. E. Valle, ler Encuentro de expertos en tecnología (págs. 86-110). Universidad Metropolitana (UNIMET).

Tejedor, J. (2008). Investigación educativa: su desarrollo en el ámbito de la Tecnología Educativa. En A. García-Valcárcel, Investigación y tecnología de la información y comunicación al servicio de la innovación educativa (págs. 177-2008). Ediciones Universidad de Salamanca.

TICSE. (2011). ¿Qué opina el profesorado sobre el Programa Escuela 2.0? Un análisis por comunidades autónomas. Informe de investigación. Obtenido de http://ntic.educacion.es/ w3//3congresoe20/Informe Escuela20-Prof2011.pdf

Trujillo, J. M et al. (2013). Educación, TIC y sociedad de la información: reflexiones, investigación y miradas críticas. Granada: Nativola. 
